\title{
Design of a Multi-layer Lane-Level Map for Vehicle Route Planning
}

\author{
Chaoran Liu, Kun Jiang, Diange Yang and Zhongyang Xiao
}

State Key Laboratory of Automotive Safety and Energy, Collaborative Innovation Center of Intelligent NewEnergy Vehicle, Department of Automotive Engineering, Tsinghua University, Beijing 100084, China

\begin{abstract}
With the development of intelligent transportation system, there occurs further demand for high precision localization and route planning, and simultaneously the traditional road-level map fails to meet with this requirement, by which this paper is motivated. In this paper, the three-layer lane-level map architecture for vehicle path guidance is established, and the mathematical models of road-level layer, intermediate layer and lane-level layer are designed considering efficiency and precision. The geometric model of the lane-level layer of the map is characterized by Cubic Hermite Spline for continuity. A method of generating the lane geometry with fixed and variable control points is proposed, which can effectively ensure the accuracy with limited number of control points. In experimental part, a multi-layer map of an intersection is built to validate the map model, and an example of a local map was generated with the lane-level geometry.
\end{abstract}

\section{Introduction}

Digital maps play an important role in current vehicle applications, e.g. vehicle localization and route planning. With more attention on intelligent transportation system, advanced driving assistance system and even driverless vehicle, the improvements in accuracy and richness are required in digital map technology [1]. When it is available to determine the vehicles' position precisely in lane level, there are a lot of probable benefits we may obtain, i.e. the transportation officials and researchers may determine distinctions in traffic conditions for different lanes on a freeway via probe vehicles [2].

Most of existing digital maps are based on road level data, which may illustrate basic information and provide useful applications for users but ignore some precise details that are essential for vehicles' high precision localization and path guidance. The improvements of accurate localization sensors such as RTK GPS and other on-board sensors such as IMU and LiDAR made the enhanced maps become possible. On the purpose of increasing utility of digital maps in advanced vehicle applications, creating the lane-level map is becoming a prevailing interest among researchers worldwide [3]. Compared with the traditional road level map, the lane level map is enhanced with massive data, which are capable of improving the accuracy from about 10 meter to decimetre level or even centimetre level and illustrating more precise geometry in line with the real situations.

The map model is supposed to be created for routing planning before geometrical representation because vehicle navigation is established on a network consisting of vertices (e.g., intersections) and edges (e.g., roads, lanes). Thus, the map model is supposed to be an abstract representation of the map database [4]. In terms of map model, there are a number of map standards containing specific explanations on the map model which we can refer to. In traditional road level map, GDF, KiW i, Navteq are mainstream dig ital map standards which have been employed widespread for decades [5], however, these road-level model are not directly applicable to lane level. RNDF is the road network definition file designed for DARPA urban challenge, in which the basic structure segment-lane-waypoint are included to provide basic information to driverless vehicles [6]. Nowadays, OpenDRIVE and NDS are well-known map standard providers, but their complicated map model and inaccessibility to public (NDS) cause difficulties for practical uses. Besides the standards above, Qing Zhu et al. [7] establish a 3-D road network which is composed of three parts: roadway centerline, carriageway, and lane. Tao Zhang et al. [4] design a lane-level map model including details on intersections and lanes. Jiang Liu et al. [8] generate enhanced intersection model and use circular to illus trate the virtual lane inside the intersection. Thus, few studies are able to provide an applicable map model with completeness and simplicity for route planning.

Another significant issue about lane-level maps is the geometrical representation of the lanes. Expanding from traditional maps, some lane illustrations in enhanced maps continue using the polyline [9]. Du Jie et al. [2] employ the piecewise polyline to approximate the centreline in a lane. Betaille et al. [3] in their lane-level enhanced map apply the clothoids for the description of the road lanes in a digital map. Circular arc spline is also applied in the map geometrical model [10]. Compared to 
there methods mentioned, Cubic Hermite Spline (CHS) may possess some advantages, because it can generate various lines, such as, straight lines, arcs, and even clothoids [11], there fore the whole map would share one type of line to accurately generate the geometrical shapes of different lanes. And there have been several studies employing the CHS to describe the shape of lanes, however, the method mentioned in [4] applies CHS to the whole lanes and even virtual lanes in the intersections without distinguishing the control points with different functions.

This paper is organized as below. Firstly, in section 2, the multi-layer lane-level map model is proposed, which includes three layer and other significant details. Then a method of geometrical representation is established by applying the CHS. Finally, the experimental validations are performed in section 4 .

\section{Multi-layer lane-level map model}

In terms of convenience of geographical illustration and efficiency of further applications like route planning, we propose a multi-layer lane-level map model, which contains three layers: road level layer, intermed iate layer, and lane level layer.

Road-level layer in this map model reserves most of traditional mathematical expressions for current existing map model, containing roads and intersections, which is intended for making use of ripe routing algorithms based on road level maps. The intermediate layer acts as a bridge between the upper layer and the lower one, in which relationship between some sets is stored for routing planning and other applications. And the third layer is designed to express the lane-level details, not only the lane sets on a road and more detailed inters ections, but also geometrical elements such as highprecision points on the centre line of a lane, lane lines and so on. An example is Figure 1

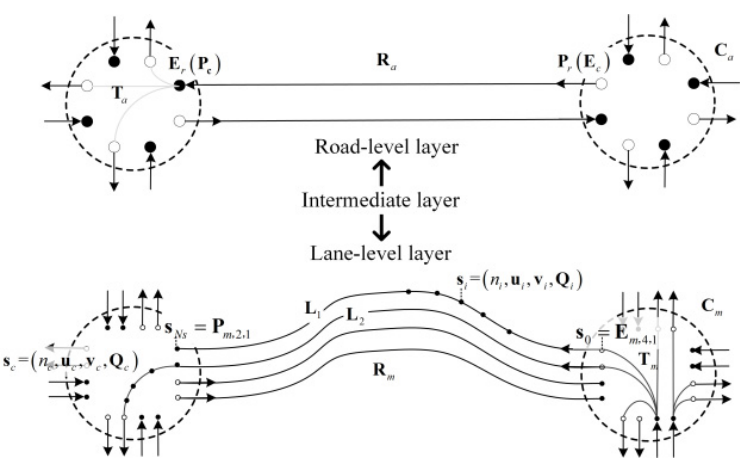

Figure 1. Multi-layer map model

\subsection{Road-level layer}

According to intuition that a road network is supposed to include roads and intersections, thus, a road-level layer is expressed as follow,

$$
\mathbf{W}_{a}=\left(\mathbf{C}_{a}, \mathbf{R}_{a}\right)
$$

where, $\mathbf{W}_{a}$ is the whole road-level network, $\mathbf{C}_{a}$ is the set of road-level intersections, and $\mathbf{R}_{a}$ is the set of roads.

The road-level intersection is defined as,

$$
\mathbf{c}_{a}=\left(\mathbf{P}_{c}, \mathbf{E}_{c}, \mathbf{T}_{a}\right)
$$

where, $\mathbf{P}_{c}$ is the set of road-level nodes entering this intersection, and $\mathbf{E}_{c}$ is the set of road-level nodes leaving this intersection. $\mathbf{T}_{a}$, road-level traffic matrix, indicating if there is a topological connection between two nodes, defined as,

$$
\begin{gathered}
P_{c, 1} \\
\mathbf{T}_{a}=\begin{array}{c}
E_{c, 1} \\
\vdots \\
E_{c, j}
\end{array}\left[\begin{array}{ccc}
\mathbf{t}_{a, 1,1} & \cdots & \mathbf{t}_{a, i, 1} \\
\vdots & \ddots & \vdots \\
\mathbf{t}_{a, 1, j} & \cdots & \mathbf{t}_{a, i, j}
\end{array}\right]
\end{gathered}
$$

where, the element $\mathbf{t}_{a, i, j}$ represents whether a vehicle could drive from the entering node $P_{c, i}$ to leaving node $E_{c, j}$, and owns mathematical expression $\mathbf{t}_{a, i . j}=\left(f_{a}, m_{a}\right)$, as shown in Figure 2, in which $f_{a}$ denotes whether $(=1)$ or not $(=0)$ a vehicle may drive from $P_{c, i}$ to $E_{c, j}$, and $m_{a}$ is the way of passing, e.g. turning left, turning right, going straight, U-turn.

The road-level road is unidirectional, which means in this model, there generally are two roads between two adjacent intersections. A road is expressed as,

$$
\mathbf{r}_{a}=\left(\mathbf{P}_{r}, \mathbf{E}_{r}, \mathbf{Q}_{a}\right)
$$

where, $\mathbf{P}_{r}$ is the set of road-level nodes entering this road, and $\mathbf{E}_{r}$ is the set of road-level nodes leaving this road. $\mathbf{Q}_{a}$ includes the road class $k_{r}$ and road length $l_{r}$.

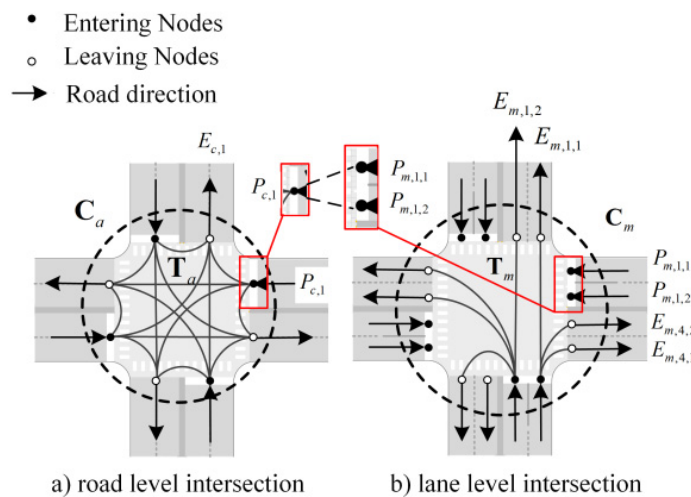

Figure 2. Traffic matrix and intermediate layer

\subsection{Intermediate layer}

In this layer, a logic connection between upper and lower layer is designed. On the purpose of providing higher precision data and conserving the advantages of traditional maps, the intermediate layer serves as a library of corresponding relationship from road level to lane level.

In an intersection $\mathbf{c}, \mathbf{P}_{c}$ is the set of its road-level incoming nodes. And without losing generality, let us assume this intersection has four road-level incoming 
nodes from different incoming directions, which could expressed as $\mathbf{P}_{c}=\left(\mathbf{P}_{c, 1}, \cdots, \mathbf{P}_{c, 4}\right)$.

And generally there are at least one lanes for each road at an intersection. As Figure 2 shows, the road-level node $\mathbf{P}_{c, 1}$ represents two lane-level nodes $\mathbf{P}_{m, 1,1}, \mathbf{P}_{m, 1,2}$.

Thus, intermediate layer is expressed as follow,

$$
\mathbf{P}_{c, i}=\left(\mathbf{P}_{m, i, 1}, \cdots, \mathbf{P}_{m, i, k_{i}}\right)
$$

\subsection{Micro layer in lane level}

Lane-level layer should not only possess more data with higher accuracy which could supplement the deficiency of road-level layer, but also provide information of complete road network for navigation.

To achieve this goal, the lane-level layer is defined as,

$$
\mathbf{W}_{m}=\left(\mathbf{C}_{m}, \mathbf{R}_{m}\right)
$$

where, $\mathbf{C}_{m}$ is the set of lane-level intersections, and $\mathbf{R}_{m}$ is the set of roads.

The road-level intersection is defined as,

$$
\mathbf{c}_{m}=\left(\mathbf{P}_{m}, \mathbf{E}_{m}, \mathbf{T}_{m}\right)
$$

where, $\mathbf{P}_{m}$ is the set of lane-level nodes entering this intersection, and $\mathbf{E}_{m}$ is the set of lane-level nodes leaving this intersection. $\mathbf{T}_{m}$, lane-level traffic matrix, indicating whether there is a topological connection between two lane-level nodes, which is defined as,

$$
\begin{gathered}
P_{m, 1} \\
\mathbf{T}_{m}=\begin{array}{c}
E_{m, 1} \\
\vdots \\
E_{m, j_{m}}
\end{array}\left[\begin{array}{ccc}
\mathbf{t}_{m, 1,1} & \cdots & \mathbf{t}_{m, i_{m}, 1} \\
\vdots & \ddots & \vdots \\
\mathbf{t}_{m, 1, j_{m}} & \cdots & \mathbf{t}_{m, i_{m}, j_{m}}
\end{array}\right]
\end{gathered}
$$

where, the element $\mathbf{t}_{m, i_{m}, j_{m}}$ implies whether and how a vehicle could drive from the entering node $P_{m, i_{m}}$ to leaving node $E_{m, j_{m}}$, and its mathematical expression is $\mathbf{t}_{m, i_{m}, j_{m}}=\left(f_{m}, m_{m}, \mathbf{S}_{c}\right)$, in which $f_{m}$ denotes whether $(=1)$ or not (=0) a vehicle may drive from $P_{m, i_{m}}$ to $E_{m, j_{m}}$, and $m_{m}$ is the way of passing which shares the same definition with that in road-level. The $\mathbf{S}_{c}$ represents the set of control points on the centre line of the virtual lane between two lane-level nodes in an intersection, as Figure 2 .

The lane-level road $\mathbf{r}_{m}$ contains all lanes on the road, thus we define,

$$
\mathbf{r}_{m}=\left(\mathbf{L}, c_{\text {in }}, c_{\text {out }}, \mathbf{Q}_{r}\right)
$$

where, $\mathbf{L}$ is the set of lanes, and $c_{\text {in }}$ and $c_{\text {out }}$ are the indices of entrance and exit intersections according to traffic directions on the road respectively, and $\mathbf{Q}_{r}$ is the attributes of the road.

Each lane in the road, i.e. element in the set of lanes, is defined as,

$$
\mathbf{I}=\left(\mathbf{S}_{l}, \mathbf{Q}_{l}\right)
$$

where, $\mathbf{S}_{l}$ is the set of control points on the centre line of the lane, and $\mathbf{Q}_{l}$ is the set of attributes, including lane width $l_{w}$, lane length $l_{e}$ and speed limit $V$.

\subsection{Node and control point}

There are nodes in road-level and lane-level (Notice that nodes in intermediate layer come from the other two layers.), which on ly exist at the connection knots between intersections and roads(lanes) and are defined as,

$$
\mathbf{N}=\left\{\mathbf{u}_{N}, \mathbf{v}_{N}\right\}
$$

where, $\mathbf{N}$ represents the entering or leaving nodes in this map model, including $\mathbf{P}_{c}, \mathbf{E}_{c}$ in the road-level intersections, $\mathbf{P}_{r}, \mathbf{E}_{r}$ in the road-level roads, and $\mathbf{P}_{m}, \mathbf{E}_{m}$ in the lane-level intersections. $\mathbf{u}_{N}$ is the location of the node, and $\mathbf{v}_{N}$ is the traffic direction of the node.

Besides nodes, control points also play an important role in layered map, especially lane-level layer. Control points in intersections and lanes both contain precise positions, tangent vectors, indices, and other attributes, and are defined as,

$$
\mathbf{S}=\left\{n_{s}, \mathbf{u}_{s}, \mathbf{v}_{s}, \mathbf{Q}_{s}\right\}
$$

where, $n_{s}$ is the serial number, and $\mathbf{u}_{s}$ is the position which is two or three-dimension vector, and $\mathbf{v}_{s}$ is the tangent vector on the node, and $\mathbf{Q}_{s}$ involves attributes, such as lateral sequence number of the lane, line types of left lane line and right lane line for control points on a lane.

Particularly, in a common scenario, the nodes at the start/end of a lane share the same positions and tangent direction with the first and the last control points.

\section{Geometrical representation}

The detailed geometry in road level is not necessary because the detailed information and precise geometry is presented in lane level and it is easy to obtain the lanelevel geometrical information $v$ ia intermediate layer from road level and on the other hand, the road level nodes are easy to locate, simple lines such as straight line could illustrate the connection and topology in road level completely. Thus, according to our multi-layer model, the lane-level geometry is discussed as below.

\subsection{Lane-level geometry}

In order to achieve the global conformity and continuity, as we mentioned before, CHS curve is employed in this part. CHS is a spline in which each segments between two control points is a three order polyline represented by Hermite form.

Assuming that two given control points are $\mathbf{s}_{i}, \mathbf{s}_{i+1}$, where $\mathbf{s}_{i}=\left(n_{i}, \mathbf{u}_{i}, \mathbf{v}_{i}\right)$, and definitions of symbols are the same with Equation (12), then the CHS generated by these two points is presented as,

$$
Q(n)=f(x)=\left[\begin{array}{c}
2 x^{3}-3 x^{2}+1 \\
x^{3}-2 x^{2}+x \\
-2 x^{3}+3 x^{2} \\
x^{3}-x^{2}
\end{array}\right]^{T}\left[\begin{array}{c}
\mathbf{u}_{i} \\
\mathbf{v}_{i} \\
\mathbf{u}_{i+1} \\
\mathbf{v}_{i+1}
\end{array}\right]
$$

where, $x=\left(n-n_{i}\right) /\left(n_{i+1}-n_{i}\right)$. 
It is easy to get the $Q\left(n_{i}\right)=\mathbf{u}_{i}, Q\left(n_{i+1}\right)=\mathbf{u}_{i+1}$, $Q^{\prime}\left(n_{i}\right)=\mathbf{v}_{i}, Q^{\prime}\left(n_{i+1}\right)=\mathbf{v}_{i+1}$, thus at each control point the first order continuity is realized, which may offer convenience for vehicle path planning due to global tangential continuity.

\subsection{Control Points Choosing}

The control points serve as the shape points in the lane level geometry, and simultaneously some of them are supposed to be feature points, of which on two sides there are some different attributes, at particular positions, e.g., the control points at the start/end of a lane. Though this kind of control points own the same form with the common control po ints, because these do not change with other variable control points, we call them the fixed control points.

\subsubsection{Fixed Control Points}

The fixed control points $\mathbf{S}_{f}$ are determined by the lane attributes. Between two adjacent fixed control points the lane attributes, except the shape of the lane, keep the same, and the lane attributes include speed limit, lateral sequence number of the lane, line types of lane lines, and etc. Some details are displayed as below.

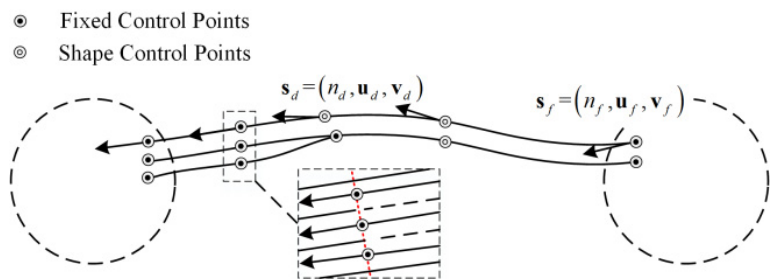

Figure 3. Control Points on lanes (Red dashed line indicates the position where the type of lane lines changes.)

\subsubsection{Variable Control Points}

The determination of shape control points occurs in two adjacent fixed control points in a lane. Assume that a series of sampled points representing the centreline of a lane are known, expressed as $\mathbf{S}_{t}=\left(\mathbf{s}_{t, 1}, \cdots, \mathbf{s}_{t, j}, \cdots, \mathbf{s}_{t, N_{t}}\right)$, and the first and last points are fixed points, i.e. $\mathbf{s}_{t, 1}, \mathbf{s}_{t, N_{t}} \in \mathbf{S}_{f}$, a stepwise approach on determining the series of shape control points $\mathbf{S}_{d}=\left(\mathbf{s}_{d, 1}, \cdots, \mathbf{s}_{d, i}, \cdots, \mathbf{s}_{d, N_{d}}\right)$ is proposed.

In this stepwise method, the distances between two adjacent shape control points are variable, which is determined by,

$$
T_{h} \geq e=\max _{j=1, \ldots, N} \min _{x \in[0,1]}\left\|\mathbf{u}_{t}(j)-f_{s_{d i,}, s_{d, i t 1}}(x)\right\|_{2}
$$

Where, $T_{h}$ is the threshold designed, which can specify the accuracy of the geometrical representation, and $e$ is the measurement of the gap between CHS curve generated and corresponding sampled point, and $\mathbf{u}_{t}(j)$ is the position extracted from $\mathbf{s}_{t, j}$ which are located among two adjacent shape control points, as shown in Figure 4.

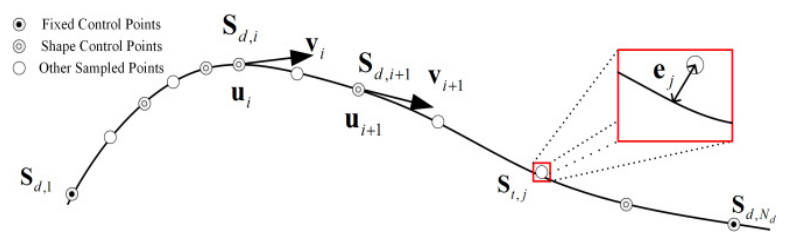

Figure 4. Shape control points choosing

The stepwise approach is started at $\mathbf{s}_{t, 1}=\mathbf{s}_{d, 1}$, then from each chosen shape control point, successively choose the next shape control point, the details are listed in Table 1.

Table 1 Pseudocode: stepwise method for shape control points

$$
\begin{aligned}
& 1 \text { set } \mathbf{S}_{d}=\left(\mathbf{s}_{t, 1}\right), \mathbf{S}_{t}=\left(\mathbf{s}_{t, 1}, \cdots, \mathbf{s}_{t, j}, \cdots, \mathbf{s}_{t, N_{t}}\right) \text {; } \\
& 2 \text { while } 1 \\
& 3 \text { if } \mathbf{S}_{t}=\varnothing \text { return } \mathbf{S}_{d} \text {; } \\
& 4 \text { set } \mathbf{S}_{d, c}=\mathbf{S}_{d}(\text { end }), k=2 \text {; } \\
& 5 \text { while } 1 \\
& 6 \quad \mathbf{S}_{d . n}=\mathbf{S}_{t}(k) \text {; } \\
& 7 \quad \mathbf{s}_{d, c}, \mathbf{s}_{d, n} \rightarrow f_{\mathbf{s}_{d, c}, \mathbf{s}_{d, n}}(x) \text {; } \\
& 8 \quad e=\max _{j=1, \ldots, k} \min _{x \in[0,1]}\left\|\mathbf{u}_{t}(j)-f_{\mathbf{s}_{d, c}, \mathbf{s}_{d, n}}(x)\right\|_{2}, \mathbf{u}_{t}(j) \in \mathbf{S}_{t}(j) ; \\
& 9 \text { if } e \geq T_{h} \text {, break; } \\
& 10 \quad \text { else } k=k+1 \text {; } \\
& 11 \quad \text { if } \mathbf{S}_{t}(k)=\varnothing \text {, break; } \\
& 12 \text { end if; } \\
& 13 \text { end if; } \\
& 14 \text { end while; } \\
& 15 \mathbf{s}_{d, n}=\mathbf{S}_{t}(k-1) \text {; } \\
& 16 \mathbf{S}_{d}=\mathbf{S}_{d} \cap \mathbf{s}_{d, n}, \mathbf{S}_{t}=\mathbf{S}_{t} /\left(\mathbf{s}_{d, c}, \cdots, \mathbf{s}_{d, n}\right) ; \\
& 17 \text { end while; }
\end{aligned}
$$

Thus, we get the complete series of control points, including fixed control points and other variable shape control points, which are combined to illustrate the lanelevel geometry.

\section{Experimental Validation}

Two experiments are conducted to validate our theoretical model and geometrical representation respectively.

In order to demonstrate the validity of multi-layer map model, we combine the aerial photography and ground calibration to establish a local map including a complete intersection which is displayed as Figure 5. The left graph is the road-level layer with the background of an aerial photo, in which the points symbolized by star are road-level nodes. The right one is the lane-level map, and the details on lanes corresponding to the road they belonging to are shown. Besides, the traffic matrix and other information are stored in $\mathrm{TAB}$ format which can be 
viewed in MapInfo and employed easily by other applications .

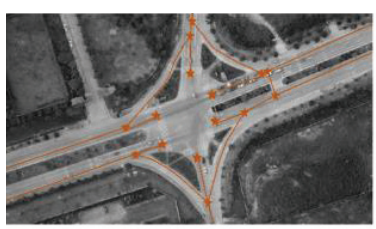

a) road-level layer in a map

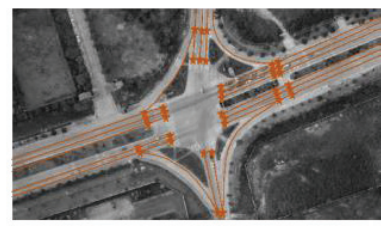

b) lane-level layer in a map
Figure 5. An example of map based on multi-layer map model

In the other experimental test, our test vehicle is equipped with RTK GPS and IMU system. The RTK GPS is BD982 from Trimble, and IMU is produced in Oxts, the model is RT2502 which can measure the position and orientation accurately. In order to measure the centreline of lanes on a road, the vehicle in our test is required to drive along the centreline as close as possible. Then the vehicle state space is established for applying Kalman Filter to obtain the precise results of locations regarded as the true value of points on centrelines. The nodes and fixed control points are determined by static vehicle with equipment running. When all the fixed lanelevel control points are measured, the stepwise method of variable shape control points is executed to pick up the other control points for lane-level geometrical representation.

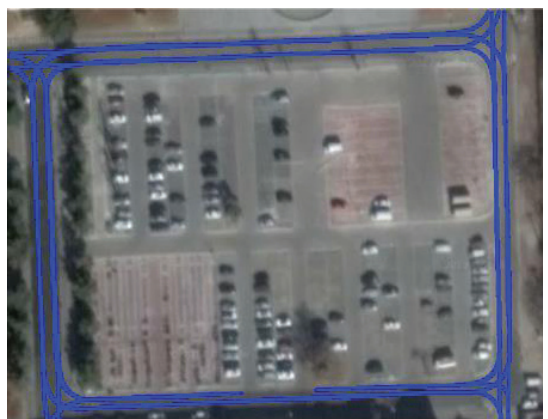

Figure 6 A lane level local map with geometry

As Figure 6 shows, the geometry representation can illustrate the shape of lanes and even intersections, increasing utility compared to the traditional geometry.

\section{Conclusions and future researches}

This paper proposes a multi-layer map model for route planning, in which we integrate the traditional road-level map with enhanced lane-level map, we also design an intermediate layer to connect the other two layers. According to advantages of CHS curve in presenting lane-level geometry, a stepwise approach to generating lanes is discussed and a geometrical system including fixed control points and shape control points is performed. A test to validate the feasibility of the map model and geometrical representation is conducted.

There are some ongoing researches based on this multi-layer map model. We have finished the route planning algorithmbased on this layered map model [12], and due to lane-level layer in this model contains all detailed information for routing, a route planning method directly employing lane-level map is also designed [13]. Then, there is a large-scale map we are building in a South-eastern province, China, which is completely based on this our layered model.

\section{Acknowledgement}

This work is supported by International Science \& Technology Cooperation Program of China under contract No.2016YFE0102200.

\section{References}

1. K. Wevers, S. Dreher. "Digital Maps for Lane Level Positioning". 15th World Congress on Intelligent Transport Systems and ITS America's 2008 Annual Meeting, (2008).

2. J. Du, M.J. Barth. "Next-generation automated vehicle location systems: positioning at the lane level". IEEE Trans. Intell. Transp. Syst. 9(1): 48-57, (2008).

3. D. Betaille, R. Toledo-Moreo. "Creating enhanced maps for lane-level vehicle navigation". IEEE Trans. Intell. Transp. Syst. 11(4):786-798, (2011).

4. T. Zhang, S. Arrigoni, M. Garozzo, et al. "A lanelevel road network model with global continuity". Transp. Res. Part C, 71:32-50, (2016).

5. T. Zhang, D. Yang, T. Li, et al. "An improved virtual intersection model for vehicle navigation at intersections," Transp. Res. Part C, 19(3):413-423, (2011).

6. J. Bohren et al., "Little Ben: The Ben Franklin Racing Team's Entry in the 2007 DARPA Urban Challenge," J. F. Robot., 25(9):598-614, (2008).

7. Q. L, Y. Zhu, "Hierarchical lane- oriented 3D road-network model," Int. J. Geogr. Inf. Sci., 22(5):479-505, (2008).

8. J. Liu, B. Cai, Y. Wang, et al. "Generating Enhanced Intersection Maps for Lane Level Vehicle Positioning based Applications," 13th COTA Int. Conf. Transp. Prof., 96(Cictp):2395-2403, (2013).

9. EDMap Consortium, "Enhanced Digital Mapping Project Final Report," (2004).

10. A. Schindler, G. Maier, F. Janda. "Generation of high precision digital maps using circular arc splines," IEEE Intell. Veh. Symp, 246-251. (2012).

11. A. Chen, A. Ramanandan, J.A. Farrell. "Highprecision lane-level road map building for vehicle navigation." IEEE Position Location and Navigation Symposium.1035-1042, (2010).

12. C. Liu, K, Jiang, Z. Xiao, Z. Cao, D. Yang, "LaneLevel Route Planning Based on a Multi-Layer Map Model." to be publis hed.

13. C. Liu, T. Zhang, S. Arrigoni, K. Jiang, F. Chelia, D. Yang, "Addressing the route planning problem on the lane-level road network," to be published. 